\title{
Loss of lag synchronization in coupled chaotic systems
}

\author{
Sosnovtseva, O.V.; Balanov, A.G.; Vadivasova, T.E.; Astakhov, V. V.; Mosekilde, Erik
}

Published in:

Physical Review E. Statistical, Nonlinear, and Soft Matter Physics

Link to article, DOI:

10.1103/PhysRevE.60.6560

Publication date:

1999

Document Version

Publisher's PDF, also known as Version of record

Link back to DTU Orbit

Citation (APA):

Sosnovtseva, O. V., Balanov, A. G., Vadivasova, T. E., Astakhov, V. V., \& Mosekilde, E. (1999). Loss of lag synchronization in coupled chaotic systems. Physical Review E. Statistical, Nonlinear, and Soft Matter Physics, 60(6), 6560-6566. https://doi.org/10.1103/PhysRevE.60.6560

\section{General rights}

Copyright and moral rights for the publications made accessible in the public portal are retained by the authors and/or other copyright owners and it is a condition of accessing publications that users recognise and abide by the legal requirements associated with these rights.

- Users may download and print one copy of any publication from the public portal for the purpose of private study or research.

- You may not further distribute the material or use it for any profit-making activity or commercial gain

- You may freely distribute the URL identifying the publication in the public portal 


\title{
Loss of lag synchronization in coupled chaotic systems
}

\author{
O. V. Sosnovtseva, ${ }^{1}$ A. G. Balanov, ${ }^{1}$ T. E. Vadivasova, ${ }^{1}$ V. V. Astakhov, ${ }^{1}$ and E. Mosekilde ${ }^{2}$ \\ ${ }^{1}$ Physics Department, Saratov State University, Astrakhanskaya Street 83, Saratov 410026, Russia \\ ${ }^{2}$ Department of Physics, The Technical University of Denmark, 2800 Lyngby, Denmark
}

(Received 21 October 1998)

\begin{abstract}
Lag synchronization denotes a particular form of synchronization in which the amplitudes of two interacting, nonidentical chaotic oscillators are correlated but there is a characteristic time delay between them. We study transitions to and between different forms of synchronization for the attractors defined as "in-phase" and "out-of-phase", and investigate the processes by which lag synchronization is lost in two coupled Rössler systems. With a small frequency mismatch between the two systems, these processes are related to the occurrence of a peculiar form of basin structure as more and more periodic orbits embedded into the synchronized chaotic state become unstable in a transverse direction. [S1063-651X(99)03711-3]

PACS number(s): 05.45.-a
\end{abstract}

\section{INTRODUCTION}

Synchronization of chaotic systems has become a significant field of research in recent years. Coupled nonlinear oscillators in chemistry [1], biology, and economics [2] are important examples. One of the exciting scientific quarries in this relation is to understand the coherent dynamical behavior of the coupled systems. In the technical realm, spread spectrum communications and systems monitoring and identification have been proposed as potential applications of chaotic synchronization, motivating further studies [3].

Several types of synchronization representing different degrees of correlation between the interacting systems have been identified and are referred to as complete (or full) synchronization [4], generalized synchronization [5], and phase synchronization $[6,7]$, respectively. Complete synchronization implies that time series of corresponding dynamical variables of the subsystems coincide completely $\mathbf{x}_{1}(t)$ $=\mathbf{x}_{2}(t)$ (i.e., the systems oscillate in phase). Generalized synchronization, as introduced for drive-response systems, is defined as the presence of some functional relationship between the states of the response and the drive, i.e., $\mathbf{x}_{2}(t)$ $=\mathcal{F}\left[\mathbf{x}_{1}(t)\right]$. For phase synchronization, suitably defined phases of two chaotic oscillators lock to each other, while their amplitudes remain uncorrelated and sustain an irregular motion of their own. Rosenblum et al. [8] have shown that for stronger coupling a new regime which is called lag synchronization may be observed. Lag synchronization appears as a coincidence of the states of two systems $\mathbf{x}_{1}(\mathbf{t}+\boldsymbol{\tau})$ $=\mathbf{x}_{2}(\mathbf{t})$ when shifted in time. With further increase of the coupling, this regime tends to complete synchronization. Lag synchronization is similar to generalized synchronization with the function $\mathcal{F}$ being defined in terms of a temporal displacement of the dynamics of the interacting subsystems.

The loss of chaotic synchronization is directly related to bifurcations of saddle periodic orbits embedded into the chaotic attractor [9-15]. The transitions to nonsynchronous behavior in systems with symmetric invariant manifolds are associated with a number of new phenomena, including riddled basins of attraction [16] and on-off intermittency [17]. The orbits embedded in the fully synchronized chaotic attractor can lose their transverse stability via a saddle- repeller pitchfork bifurcation [10], a period-doubling bifurcation [14], or a Hopf bifurcation [18]. These bifurcations may lead to attractor bubbling as described by Ashwin et al. [19]. Trajectories repelled from the synchronized chaotic state may then make an excursion out in phase space. Sooner or later, however, almost all trajectories will return to the vicinity of the symmetric subspace. When riddling occurs, a dense set of initial conditions which belong to the basin of another attractor appears within the basin of the symmetric chaotic attractor $[18,19]$. A number of issues related to the influence of asymmetry and noise were discussed in [19].

Investigations of these synchronization phenomena have often considered coupled fully identical maps as mathematical models. Results obtained in the framework of such idealizations have then been applied to explain the behavior of real systems. When detuning between the basic frequencies of the interacting oscillators is introduced, regions of chaotic phase synchronization, similar to Arnol'd tongues for periodic oscillations, appear on the parameter plane. The transition phenomena that take place at the boundary of chaotic synchronization are associated with the bifurcations of saddle periodic orbits as well. Anishchenko et al. [12] have ascribed this boundary to an accumulation of curves of tangent bifurcations of saddle cycles, and a more recent study by Pikovsky et al. [13] suggests that attractor-repeller collisions take place at the transition to chaotic synchronization, thus drawing on the analogy with the tangent bifurcation of a limit cycle. Most recently [15], the transition to phase synchronization was described as a boundary crisis mediated by unstable-unstable pair bifurcations on a branched manifold.

In this paper, considering the example of two coupled Rössler systems, we investigate the effect of a frequency mismatch on the synchronization phenomena. Transitions to and between complete, lag, and phase synchronization for different regimes are discussed. The process by which lag synchronization is lost and phase synchronization takes place is described in terms of bifurcations of periodic orbits embedded into the chaotic attractor.

\section{CHARACTERIZING THE SYNCHRONIZATION}

Synchronization is a universal nonlinear phenomenon, and many of its key features are typically independent of the 

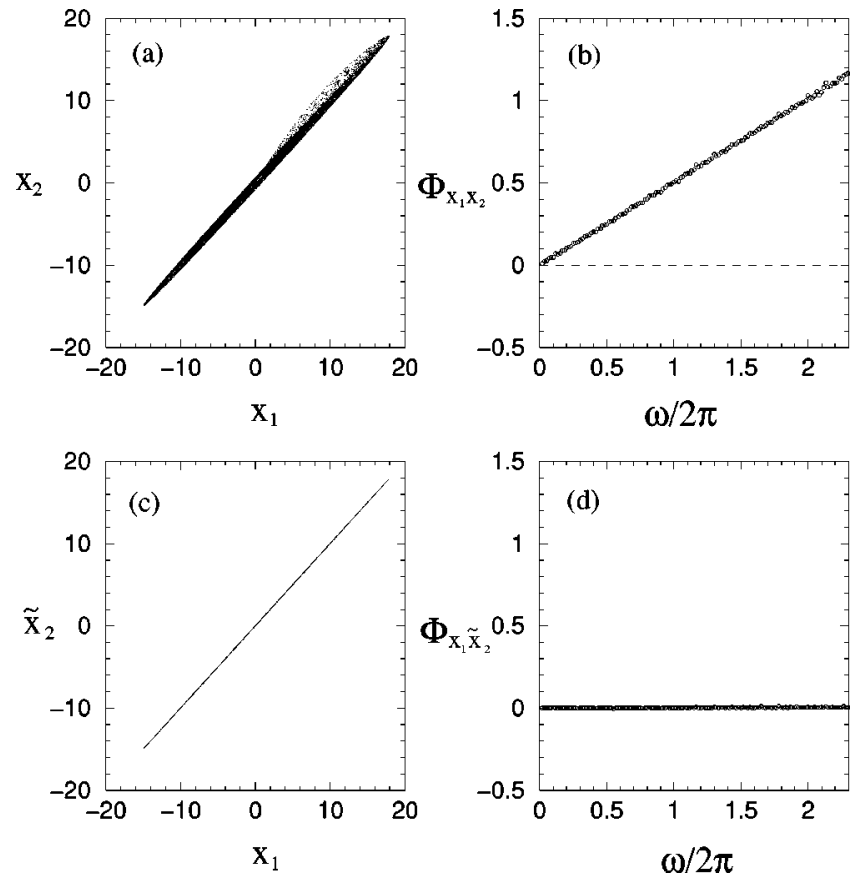

FIG. 1. Example of lag synchronization for two coupled Rössler systems $\left(\alpha=0.165, \quad \beta=0.2, \quad \omega_{0}=0.97, \quad \mu=10.0, \quad \gamma=0.2, \quad \Delta\right.$ $=0.005$ ). Projections on the plane $\left[x_{1}(t) x_{2}(t)\right]$ (a) and function $\Phi_{x_{1} x_{2}}$ vs $\omega$ (b) with similar delayed-coordinate plots $\tilde{x}_{2}$ and $\Phi_{x_{1} \tilde{x}_{2}}$ (c) and (d), respectively. $\tilde{x}_{2}=x_{2}(t+\tau)$ and $\Phi_{x_{1} \tilde{x}_{2}}=\Phi_{x_{1}(t) x_{2}(t+\tau)}$.

particular model. As an example, we consider a system of coupled Rössler oscillators as described by Rosenblum et al. [6]:

$$
\begin{gathered}
\dot{x}_{1}=-\omega_{1} y_{1}-z_{1}+\gamma\left(x_{2}-x_{1}\right), \\
\dot{y}_{1}=\omega_{1} x_{1}+\alpha y_{1}, \\
\dot{z}_{1}=\beta+z_{1}\left(x_{1}-\mu\right), \\
\dot{x}_{2}=-\omega_{2} y_{2}-z_{2}+\gamma\left(x_{1}-x_{2}\right), \\
\dot{y}_{2}=\omega_{2} x_{2}+\alpha y_{2}, \\
\dot{z}_{2}=\beta+z_{2}\left(x_{2}-\mu\right),
\end{gathered}
$$

where the parameters $\alpha, \beta$, and $\mu$ govern the dynamics of the individual subsystem. $\gamma$ is the coupling parameter, $\omega_{1}$ $=\omega_{0}+\Delta$ and $\omega_{2}=\omega_{0}-\Delta$ are the natural frequencies, and $2 \Delta$ is the mismatch between these frequencies.

The above equations serve as a good model for real systems demonstrating a period-doubling route to chaos, e.g., for electronic circuits $[12,20]$, as well as for many chemical [21] and biological [2] systems.

Since synchronization between two systems involves phase relations, the notion of the phase for chaotic oscillators and several methods to characterize the phase dynamics were intensively discussed [6-8]. For the Rössler system the instantaneous phase and amplitude can be conveniently introduced as $[7,21]$

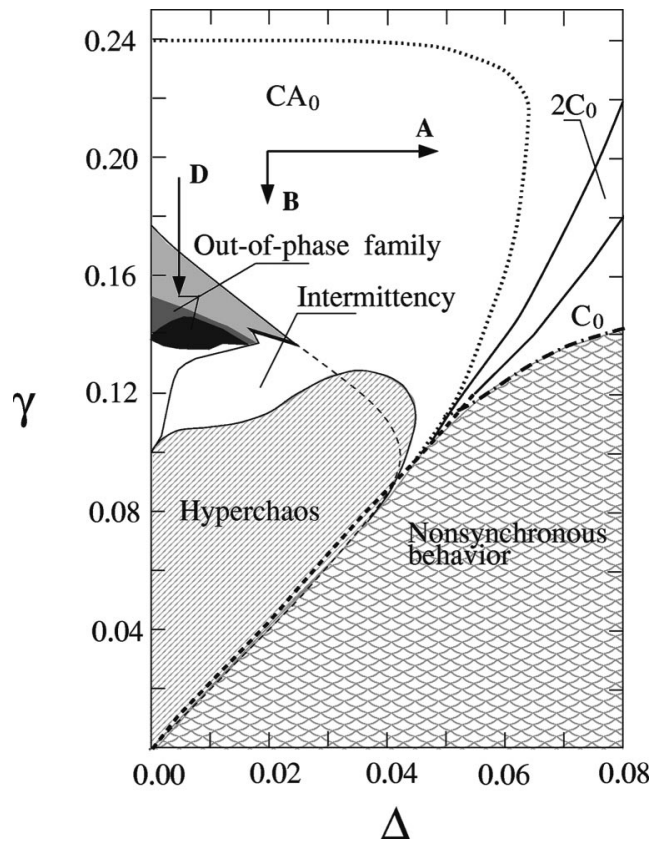

FIG. 2. Simplified bifurcation diagram for two coupled nonidentical Rössler systems $\left(\alpha=0.165, \beta=0.2, \omega_{0}=0.97, \mu=10.0\right)$. Gray-colored regions indicate transitions from periodic solutions to chaotic regimes for the out-of-phase family. Dotted curve corresponds to a period-doubling bifurcation for the saddle cycle $C_{0}$; dashed curves denote tangent bifurcations of saddle cycles; dotteddashed curve is torus birth bifurcation.

$$
\phi=\arctan \frac{y}{x}+\pi K, K=0, \pm l,+2, \ldots, \quad A=\left(x^{2}+y^{2}\right)^{1 / 2}
$$

It is easy then to define a phase difference between the interacting oscillators $\delta \phi(t)=\phi_{2}(t)-\phi_{1}(t)$. If the relation $\left|n \phi_{1}-m \phi_{2}\right|<$ const is fulfilled for some period of time, one can speak of phase locking. In many instances the weaker condition for frequency locking $\delta \Omega=\left\langle\dot{\phi}_{1}-\dot{\phi}_{2}\right\rangle=0$ is used. Here, \langle\rangle denotes time average and $\delta \Omega$ is the difference between the mean angular frequencies. $m$ and $n$ are integers.

To describe the lag synchronization, we have to introduce some relevant measures. An appropriate quantity to characterize the time shift in the lag synchronized regime is the cross-spectral density [22]:

$$
G_{u \mathrm{v}}(\omega)=\left|G_{u \mathrm{v}}(\omega)\right| e^{j \Phi_{u \mathrm{v}}(\omega)}=\int_{-\infty}^{\infty} R_{u \mathrm{v}}(\tau) e^{-j \omega \tau} d \tau
$$

where $R_{u \mathrm{v}}(\tau)=\langle[u(t)-\langle u(t)\rangle][\mathrm{V}(t-\tau)-\langle\mathrm{v}(t)\rangle]\rangle$ is the cross-correlation function for $u(t)$ and $\mathrm{V}(t)$. If $u(t)$ and $\mathrm{v}(t)$ satisfy the condition $\mathrm{V}(t)=u(t-\tau)$, then

$$
\Phi_{u \mathrm{v}}(\omega)=\arg \left(G_{u \mathrm{v}}(\omega)\right)=\omega \tau
$$

This implies that time lag $\tau=\Phi_{u \mathrm{v}}(\omega) / \omega$.

The phenomenon of lag synchronization is clearly demonstrated in Fig. 1. Here, we have plotted $x_{2}(t)$ versus $x_{1}(t)$ (a) and $\Phi_{x_{1} x_{2}}$ versus $\omega$ (b). We observe how the monotonically growing function $\Phi_{x_{1} x_{2}}$ remains a straight line within 

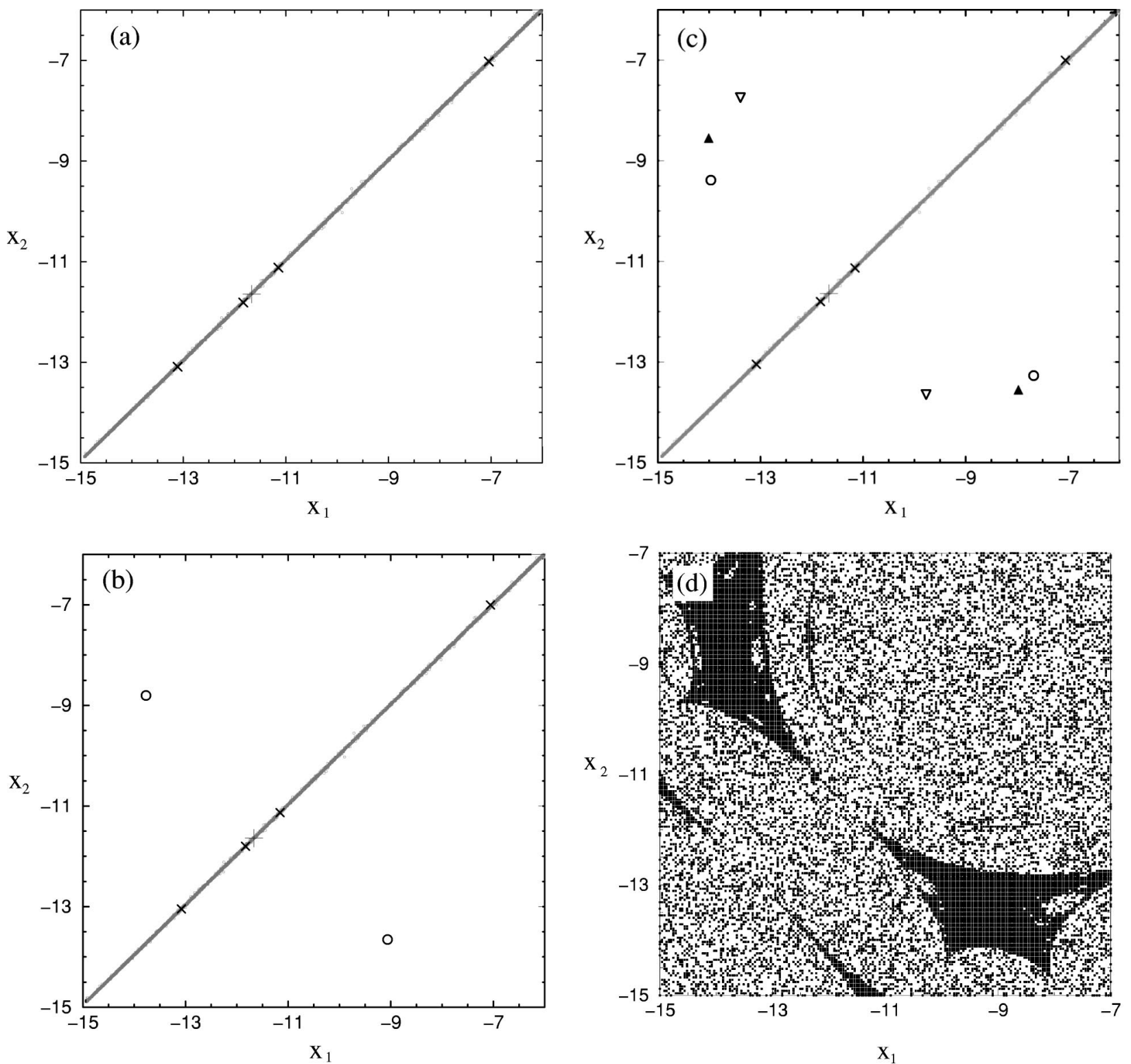

FIG. 3. (a) The saddle cycle $C_{0}(+)$, and the saddle $4 C_{0}(\times)$ embedded into the chaotic set $C A_{0} \quad(\gamma=0.25)$; (b) the saddle $2 C_{1}^{s}$ ( $\left.\bigcirc\right)$ that appears from $C_{0}$ via a period-doubling transition $(\gamma=0.3)$; (c) the saddles $2 C_{1}^{s}(\bigcirc), 2 C_{1}^{n}(\nabla)$, and stable cycle $2 C_{1}(\boldsymbol{\Delta})$ after a saddlenode bifurcation $(\gamma=0.16)$; and $(\mathrm{d})$ the riddledlike structure $(\gamma=0.1437)$. Black dots belong to the basin of the stable quasiperiodic regime $2 T_{1}\left(\alpha=0.165, \beta=0.2, \omega_{0}=0.97, \mu=10.0, \Delta=0.005\right)$.

the accuracy of the calculation. This allows us to determine the slope and, hence, the time shift $\tau$. Figure 1 also shows the reconstructed attractor, i.e., $\tilde{x}_{2}=x_{2}(t+\tau)$ versus $x_{1}(t)$ in the new symmetric subspace $x_{1}=\tilde{x}_{2}$ (c) as well as the corresponding delayed-coordinate function $\Phi_{x_{1} \tilde{x}_{2}}=\Phi_{x_{1}(t) x_{2}(t+\tau)}$ (d). Outside the region of lag synchronization, while still increasing on the average with $\omega$, the function $\Phi_{x_{1} x_{2}}$ will oscillate with some amplitude. Hence, a unique slope cannot be defined, and the fully symmetric synchronized attractor cannot be constructed. Numerically obtained time shifts prove the states of the subsystems to be identical but shifted in time with respect to each other. These results are in agreement with results reported by Rosenblum et al. [8].

\section{DYNAMICS INSIDE THE REGION OF SYNCHRONIZATION}

\section{A. The set of attractors}

Many processes in nature are characterized by the coexistence of a large number of attractors for a fixed set of parameters but attainable from different initial conditions. In the presence of weak interaction, the phenomenon of multistability can be observed in coupled systems which individually possess only one attractor at fixed parameter values [2325]. For two synchronized oscillators whose spectrum contains subharmonics $\omega_{0} / 2^{k}(k=1,2,3, \ldots)$ of the basic frequency, the phase difference between interacting units can attain $2^{k}$ different values, i.e., $\delta \phi=2 \pi l, l=0,1,2, \ldots, 2^{k}$ 

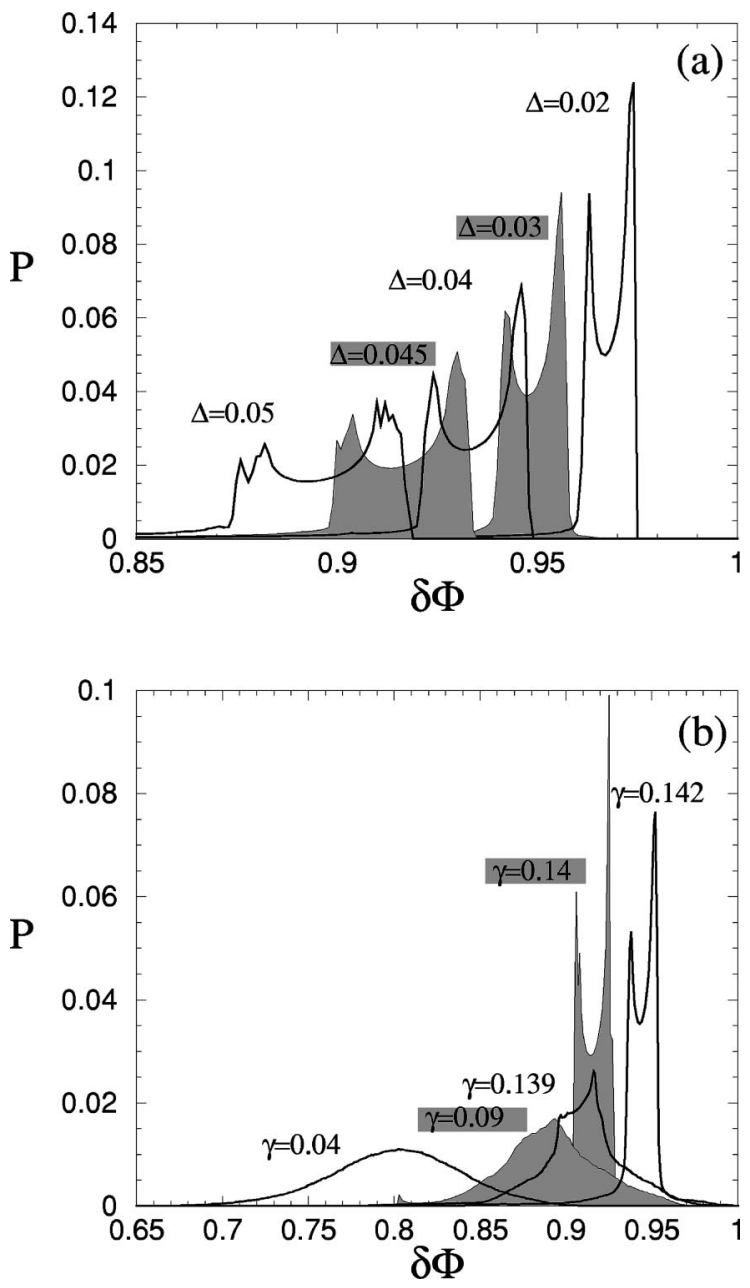

FIG. 4. The distribution $P$ of momentary phase differences (a) at $\gamma=0.205$ along the direction $A$ in Fig. 2 for $\Delta=0.02 ; 0.03 ; 0.04$; $0.045 ; 0.05$ (from the right to the left) and (b) at $\Delta=0.02$ along the direction $B$ in Fig. 2 for $\gamma=0.142 ; 0.14 ; 0.139 ; 0.09 ; 0.04$ (from the right to the left) $\left(\alpha=0.165, \beta=0.2, \omega_{0}=0.97, \mu=10.0\right)$.

-1 . Hence, a large number of periodic and chaotic attractors can coexist within a wide range of parameters. We start our study from the case of identical systems $(\Delta=0)$. Let us consider attractors from only two families: "in-phase"' attractors for which the phase difference of $x_{1}(t)$ and $x_{2}(t)$ is zero and "out-of-phase" attractors where the phase difference is $2 \pi$. Denote the periodic attractors as $2{ }^{k} C_{0}$ and $2{ }^{k} C_{1}$ and the corresponding chaotic attractors as $2^{i} C A_{0}$ and $2^{i} C A_{1}$, respectively. Here $2^{k}(k=1,2,3, \ldots)$ represents the cycle period, normalized in terms of the single period- 1 solution $C_{0}$ which originally exists in the system; $2^{i}(i=0,1,2, \ldots)$ represents the number of bands for the chaotic attractors.

With $\Delta=0$, as $\mu$ is increased the system (1) demonstrates a cascade of period-doubling bifurcations of periodic orbits $2^{k} C_{0}$ leading to the formation of a chaotic attractor in the symmetric subspace. With further increase of $\mu$, bandmerging bifurcations of the chaotic attractors $2^{i} C A_{0}$ take place. All of these attractors are located in the symmetric subspace of the whole phase space of the system. This corresponds to the case of complete synchronization. The mutual entrainment of periodic oscillations $2 C_{1}$ which is symmetric under the coordinate transformation $\left(x_{1}, x_{2}\right) \leftrightarrow\left(x_{2}, x_{1}\right) ; \quad\left(y_{1}, y_{2}\right) \leftrightarrow\left(y_{2}, y_{1}\right) ; \quad\left(z_{1}, z_{2}\right) \leftrightarrow\left(z_{2}, z_{1}\right)$ can be viewed as a particular case of lag synchronization corresponding to a time shift of half a period between the above states. A sequence of bifurcations of this attractor leads to the appearance of high-periodic solutions and chaotic attractors $2^{i} C A_{1}$ which are phase locked but situated outside the symmetric subspace.

For the nonidentical case $(\Delta \neq 0)$, Anishchenko et al. [26] have shown that regions of chaotic synchronization such as Arnol'd tongues exist. Inside these regions a gradual transition from complete to lag synchronization for "in-phase" attractors takes place. "Out-of-phase"' solutions demonstrate phase coherence properties, but for these solutions full coherence cannot be achieved. Moreover, "out-of-phase", attractors seem to remain structurally stable over a longer interval of frequency mismatch. Hence, the transition to nonsynchronous behavior is determined by the loss of stability for the "out-of-phase", synchronized mode.

\section{B. Transition from lag to phase synchronization}

\section{The case of a small mismatch}

Let us consider how the set of attractors reconstructs itself in the process of loss of lag synchronization when the coupling is changed. Figure 2 shows the bifurcation diagram for the synchronous solution on the $(\Delta, \gamma)$ parameter plane. Throughout this section the symmetric subspace is considered in lag coordinates, that is, $x_{1}(t)=x_{2}(t+\tau)$.

With a small mismatch, the boundary of chaotic phase synchronization is located in the region of accumulation of curves of tangent bifurcations of saddle cycles (family of dashed curves in the lower left corner of Fig. 2). At high values of the coupling strength there exists the regime of lag synchronization which is stable to perturbations. With decreasing of the coupling, this state becomes sensitive to small intensity noise. Excursions from the "synchronous" attractor is observed. Then bursts of this behavior gradually become stronger and are observed even without noise. At this moment lag synchronization is no longer observed. When coupling is further decreased, the second Lyapunov exponent becomes positive. Hence, a transition to hyperchaos occurs. We are interested in the bifurcations that cause these transitions.

Let us consider the mechanism of loss of synchronization from the point of view of bifurcations of saddle periodic orbits embedded into the chaotic attractor. We fix the mismatch between the basic frequencies at $\Delta=0.005$ and investigate the transitions between the various regimes when the coupling strength is decreased (the direction $D$ in Fig. 2). The periodic orbits $C_{0}$ and $2^{k} C_{0}$ are of saddle type and determine the structure of $C A_{0}$ [Fig. 3(a)]. At $\gamma=0.239$ (dotted curve in Fig. 2) the saddle cycle $C_{0}$ undergoes a perioddoubling bifurcation. As a result, $C_{0}$ loses its stability in a direction transverse to the synchronization manifold and a saddle period-2 cycle $2 C_{1}^{s}$ softly appears in its vicinity. When the coupling is further decreased, the points in the Poincare section of this cycle move away from the symmetric subspace [Fig. 3(b)]. At $\gamma=0.1657$, a saddle-node bifurcation (boundary of the light gray region in Fig. 2) takes place leading to the formation of a stable asymmetric period- 2 cycle. In the neighborhood of the saddle periodic orbit $2 C_{1}^{S}$ the stable periodic orbit $2 C_{1}$ and a saddle periodic 
orbit $2 C_{1}^{n}$ appear [Fig. 3(c)]. From some initial conditions near the chaotic attractor $C A_{0}$, the trajectory converges to $2 C_{1}$. However, the length of this transient is very sensitive to the initial conditions. When this happens, a basin of attraction of phase trajectories by $2 C_{1}$ is formed. The immediate basin has a tonguelike shape and is bounded by the stable manifolds of the saddle cycles $2 C_{1}^{s}$ and $2 C_{1}^{n}$.

At lower values of the coupling, a similar transverse destabilization takes place for other periodic cycles such as $4 C_{0}$ and $8 C_{0}$ and several of these subsequently undergo a saddle-node bifurcation. In this way, a set of tongues appears step by step and the basin of attraction of $C A_{0}$ becomes riddled by initial conditions from which trajectories move to the stable regime out of the symmetric subspace [Fig. 3(d)]. At $\gamma=0.1443$, a torus develops from $2 C_{1}$ (dark gray region in Fig. 2). At this moment, noise of small intensity induces the escape from attractor $C A_{0}$. When the coupling is further decreased, $C A_{1}$ appears via bifurcations of resonant cycles (the black region in Fig. 2) to subsequently become unstable in a boundary crisis. Chaos-chaos intermittency between $C A_{0}$ and $C A_{1}$ then is observed, and lag synchronization is lost. The chaotic attractor $C A_{0}$ and the chaotic saddle set merge, and hyperchaos arises.

A similar sequence of bifurcations is observed in a wide range of frequency mismatch (until $\Delta=0.01$ ). At these values of $\Delta$, a tangent bifurcation of the higher periodic cycles $\left(4 C_{1}\right.$ and $\left.8 C_{1}\right)$ takes place and chaos $C A_{1}$ appears via a sequence of period doubling bifurcations.

\section{The case of a large mismatch}

At large values of the frequency mismatch the region of synchronization is bounded downwards by the curve of torus birth bifurcation where two complex-conjugate multipliers cross the unit circle (dotted-dashed curve in Fig. 2). Following the lag synchronized attractor, one observes that this is gradually transformed into the phase-synchronized attractor $C A_{0}$. Figure 4(a) shows how the distribution of instantaneous phase differences changes gradually when the frequency mismatch is increased (direction $A$ in Fig. 2). The rightmost distribution with the highest maxima and the smallest widths corresponds to $\Delta=0.02$, and the other distributions with lower maxima and broader width corresponds to $\Delta=0.03,0.04,0.045$, and 0.05 . But for any frequency mismatch there is a coupling interval where lag synchronization occurs. This is to be compared with the case where the lag synchronized regime is immediately destroyed (direction $B$ in Fig. 2). Under these conditions the distribution of phase differences abruptly changes its form when chaos-chaos intermittency appears at $\gamma=0.139$ [Fig. 4(b)].

Note that, in contrast to the case of a weak mismatch when frequency (and phase) locking takes place, synchronization in the case of a large mismatch is related to the suppression of oscillations of one of the coupled subsystems by the signal of the other subsystem. Therefore, the evolution of the lag attractor is different in the two cases, but this question needs further investigations.

\section{CONCLUSIONS}

In this paper we have studied the effect of mutual synchronization of self-sustained oscillations in coupled Rössler systems whose natural frequencies are different. Based on the results of computer simulation, we demonstrated that a regime of complete synchronization for "in-phase" attractors is transferred into a lag regime when a frequency mismatch is introduced while "out-of-phase" attractors remain phase synchronized. With decreasing coupling, the loss of lag synchronization is related to a sequence of bifurcations of saddle cycles embedded into the chaotic attractor and to bifurcations of "out-of-phase" attractors. Reconstructing the lag synchronized attractor, we found an analogy to the loss of complete synchronization via a riddling transition for the case of identical oscillators. We believe that different forms of synchronization which are related to different attracting sets coexisting in a parameter space of a system can find a number of practical applications in description, for example, of biological systems that contain a large number of complex oscillators.

\section{ACKNOWLEDGMENTS}

This work was supported in part by RFFI (Grant No. 9802-16531) and INTAS (Grant No. 96-0305). O.S. and A.B. acknowledge support from the Danish Research Academy, and T.E. acknowledges support from ISSEP (Grant No. d99835).
[1] Y. Kuramoto, Chemical Oscillations, Waves and Turbulence (Springer, Berlin, 1984).

[2] E. Mosekilde, Topics in Nonlinear Dynamics. Applications to Physics, Biology and Economics Systems (World Scientific, Singapore, 1996).

[3] K. M. Cuomo and A. V. Oppenheim, Phys. Rev. Lett. 71, 65 (1993); T. L. Carroll and L. M. Pecora, Physica D 67, 126 (1993); L. Kosarev and U. Parlitz, Phys. Rev. Lett. 74, 5028 (1995); R. Brown, N. F. Rul'kov, and N. B. Tufillaro, Phys. Lett. A 196, 206 (1994).

[4] H. Fujisaka and T. Yamada, Prog. Theor. Phys. 69, 32 (1983); L. Pecora and T. Carroll, Phys. Rev. Lett. 64, 821 (1990); A. S. Pikovsky, Z. Phys. B: Condens. Matter 55, 149 (1984).

[5] N. F. Rulkov, M. M. Suschik, L. S. Tsimring, and H. D. I. Abarbanel, Phys. Rev. E 51, 980 (1995); L. Kosarev and U.
Parlitz, Phys. Rev. Lett. 76, 1816 (1996).

[6] M. G. Rosenblum, A. S. Pikovsky, and J. Kurths, Phys. Rev. Lett. 76, 1804 (1996).

[7] A. S. Pikovsky, M. G. Rosenblum, G. V. Osipov, and J. Kurths, Physica D 104, 219 (1997).

[8] M. G. Rosenblum, A. S. Pikovsky, and J. Kurths, Phys. Rev. Lett. 78, 4193 (1997).

[9] A. S. Pikovsky and P. Grassberger, J. Phys. A 24, 4587 (1991).

[10] Y.-C. Lai, C. Grebogi, J. A. Yorke, and S. C. Venkataramani, Phys. Rev. Lett. 77, 55 (1996).

[11] N. F. Rulkov and M. M. Suschik, Phys. Lett. A 214, 145 (1996).

[12] V. S. Anishchenko, T. E. Vadivasova, D. E. Postnov, and M. A. Safonova, Int. J. Bifurcation Chaos Appl. Sci. Eng. 2, 633 (1992). 
[13] A. Pikovsky, G. Osipov, M. Rosenblum, M. Zaks, and J. Kurths, Phys. Rev. Lett. 79, 47 (1997).

[14] V. Astakhov, A. Shabunin, T. Kapitaniak, and V. Anishchenko, Phys. Rev. Lett. 79, 1014 (1997).

[15] E. Rosa, Jr., E. Ott, and M. H. Hess, Phys. Rev. Lett. 80, 1642 (1998).

[16] J. C. Alexander, J. A. Yorke, Z. You, and I. Kan, Int. J. Bifurcation Chaos Appl. Sci. Eng. 2, 795 (1992); J. C. Alexander, B. R. Hunt, I. Kan, and J. A. Yorke, Erg. Theoret. Dyn. Syst. 16, 651 (1996); J. C. Sommerer and E. Ott, Nature (London) 365, 136 (1993); E. Ott and J. C. Sommerer, Phys. Lett. A 188, 39 (1994).

[17] N. Platt, E. A. Spiegel, and C. Tresser, Phys. Rev. Lett. 70, 279 (1993).

[18] Yu. L. Maistrenko, V. L. Maistrenko, A. Popovich, and E. Mosekilde, Phys. Rev. E 57, 2713 (1998); T. Kapitaniak, Yu. Maistrenko, A. Stefanski, and J. Brindley, ibid. 57, 6253 (1998); S. Yanchuk, Yu. Maistrenko, and E. Mosekilde (unpublished).

[19] P. Ashwin, J. Buescu, and I. N. Stewart, Nonlinearity 9, 703
(1996); S. C. Venkataramani, B. R. Hunt, and E. Ott, Phys. Rev. E 54, 1346 (1996); V. Astakhov, M. Hasler, T. Kapitaniak, A. Shabunin, and V. Anishchenko, ibid. 58, 5620 (1998).

[20] J. F. Heagy, T. L. Caroll, and L. M. Pecora, Phys. Rev. E 50, 1874 (1994).

[21] A. Goryachev and R. Kapral, Phys. Rev. Lett. 76, 1619 (1996).

[22] J. S. Bendat and A. G. Piersol, Random Data (John Wiley \& Sons, New York, 1986).

[23] V. V. Astakhov, B. P. Bezruchko, E. N. Erastova, and E. P. Seleznev, J. Tekh. Fiz. 60, 19 (1990) [Sov. Phys. Tech. Phys. 35, 1122 (1990)].

[24] D. E. Postnov, T. E. Vadivasova, O. V. Sosnovtseva, A. G. Balanov, V. S. Anishchenko, and E. Mosekilde, Chaos 9, 227 (1999).

[25] J. Rasmussen, E. Mosekilde, and C. Reick, Math. Comput. Simul. 40, 247 (1996).

[26] V. S. Anishchenko, V. V. Astakhov, T. E. Vadivasova, O. V. Sosnovtseva, and L. O. Chua, Int. J. Bifurcation Chaos Appl. Sci. Eng. 5, 1677 (1995). 